\title{
Enhanced transdermal bioavailability of testosterone propionate via surfactant-modified ethosomes
}

This article was published in the following Dove Press journal:

International Journal of Nanomedicine

12 August 2013

Number of times this article has been viewed

\author{
Shu Meng' \\ Zaixing Chen ${ }^{2}$ \\ Liqun Yang' \\ Wei Zhang' \\ Danhua Liu' \\ Jing Guo' \\ Yanmin Guan' \\ Jianxin $\mathrm{Li}^{\prime}$ \\ 'Liaoning Research Institute of Family \\ Planning, Shenyang, Liaoning Province, \\ People's Republic of China; ${ }^{2}$ School of \\ Pharmacy, China Medical University, \\ Shenyang, Liaoning Province, People's \\ Republic of China
}

Correspondence: Jianxin Li Liaoning Research Institute of Family Planning, 10 Puhe Street, Shenyang, Liaoning Province, People's Republic of China, II 1003 I

Tel +88602426 II 2802

Fax +88 602486806307

Email jxinl@vip.sina.com

\begin{abstract}
The current investigation aimed to evaluate the transdermal potential of novel testosterone propionate (TP) ethosomes and liposomes prepared by surfactant modification. The effect of hexadecyl trimethyl ammonium bromide and cremophor EL-35 on the particle size and zeta potential of the prepared vesicles was investigated. The entrapment efficiency and stability, as well as in vitro and in vivo skin permeation, were studied with the various techniques, such as differential scanning calorimetry, confocal laser scanning microscopy, transmission electron microscopy, dynamic light scattering, and so on. The results indicated that the ethosomes were defined as spherical, unilamellar structures with low polydispersity $(0.100 \pm 0.015)$ and nanometric size $(156.5 \pm 3.5 \mathrm{~nm})$. The entrapment efficiency of TP in ethosomal and liposomal carriers was $92.7 \% \pm 3.7 \%$ and $64.7 \% \pm 2.1 \%$, respectively. The stability profile of the prepared TP ethosomal system assessed for 120 days revealed very low aggregation and very low growth in vesicular size. TP ethosomes also provided an enhanced transdermal flux of $37.85 \pm 2.8 \mu \mathrm{g} / \mathrm{cm}^{2} /$ hour and a decreased lag time of 0.18 hours across mouse skin. The skin permeation efficiency of the TP ethosomes as further assessed by confocal laser scanning microscopy revealed enhanced permeation of rhodamine red-loaded formulations to the deeper layers of the skin $(260 \mu \mathrm{m})$ than that of the liposomal formation $(120 \mu \mathrm{m})$.
\end{abstract}

Keywords: testosterone propionate, surfactant-modified ethosomes, liposomes, confocal laser scanning microscopy

\section{Introduction}

Testosterone not only plays an important role for men, but also for women. Its biological effects extend beyond the reproductive system, and are almost ubiquitous throughout the nonreproductive systems, although it exerts these effects in different ways and by different mechanisms. A physiological decrease in the testosterone level in men causes various changes with clinical significance, such as developmental delay in youths, climacteric syndrome, and inner secretory erectile dysfunction (impotence); meanwhile, testosterone is a very important hormone for postmenopausal women, especially in terms of staying fit and maintaining energy and sexual functioning. Testosterone also appears to exert vasomotor effects in the vagina, enhancing vaginal blood flow and lubrication. Moreover, testosterone may affect many body systems and functions, including blood, body calcium balance and bone mineralization, lipid and sugar metabolism, and prostate growth. ${ }^{1}$ With so many relevant biological properties, testosterone has significant therapeutic potential. Recent testosterone replacement therapy has primarily been based on oral, intramuscular, and transdermal preparations..$^{2-4}$ Direct injection of testosterone is very painful. Additionally, testosterone has a short plasma half-life $\left(\mathrm{t}_{1 / 2}<2-4\right.$ hours $)$, so most people 
are not willing to undergo direct injection of pure testosterone each day. Because of these factors, esterification has been adopted to produce many testosterone-esters; eg, testosterone propionate (TP), testosterone enanthate, testosterone cypionate, and testosterone undecanoate. These testosterone esters may extend the half-life of testosterone to different degrees and reduce the pain of injection. Currently, they are the main forms of injections used in the clinic..$^{5,6}$ However, intramuscular injections often yield supra- and subphysiological testosterone levels.

In recent years, the ethosome has been developed as a new drug carrier with high deformability, high entrapment efficiency, and a good transdermal permeation rate in a drug-delivery system that is suitable for transdermal administration..$^{7-11}$ Ethosomes are composed mainly of phospholipids, with a relatively high concentration of ethanol, water and some surfactants. ${ }^{12-14}$ Furthermore, ethosomes are also able to provide an effective intracellular delivery of both hydrophilic and lipophilic molecules. ${ }^{15}$

In this work, transdermal testosterone propionate ethosomes and liposomes were designed and modified to determine which one could deliver the drug into the skin most efficiently. An in vivo animal study was conducted to evaluate the depth of the skin penetration based on determining the concentration of Rhodamine red-X and succinimidyl ester (RR) by CLSM. The goal of this study was to develop a sustained-release dermal delivery with a stable formulation and good entrapment efficiency, release rate, and transdermal absorption.

\section{Materials and methods Materials}

Soybean phosphatidylcholine (PC) was purchased from Shanghai Taiwei Pharmaceutical Industry Ltd, (Shanghai, People's Republic of China), and contained 98\% phosphatidylcholine. Phosphotungstic acid (PTA) was purchased from Sigma-Aldrich Corporation (St Louis, MO, USA). Rhodamine red-X and succinimidyl ester (RR) was purchased from Invitrogen Corporation (Eugene, Oregon, USA). TP was purchased from Wuhan Yuancheng Pharmaceutical Ltd., (Wuhan, People's Republic of China), Cremophor EL-35 was purchased from BASF (Ludwigshafen, Germany). Hexadecyl trimethyl ammonium bromide (CTAB) was purchased from Beijing aoboxing Biotechnology Co, Ltd., (Beijing, People's Republic of China). Methanol and tetrahydrofuran were of chromatographic grade. All other chemicals used throughout this investigation were of analytical grade, and no additional purification was performed. Triple-distilled water was used wherever required.

\section{TP solubility measurements}

TP solubility was measured in water and aqueous solutions of sodium dodecyl sulfate (SDS), Tween-80, polyethylene glycol-400 (PEG400), and Cremophor EL-35 at $37^{\circ} \mathrm{C}$. Briefly, an excess of TP was added to $10 \mathrm{~mL}$ of solvent, and the suspensions were vigorously shaken with HNY-1102C incubator shaker (Tianjin Honor Instrument Co, Tianjin, People's Republic of China) for 72 hours until a stable sediment was obtained. ${ }^{16}$ Samples were centrifuged for 10 minutes at 5,000 rpm, and the supernatant was filtered (Millipore $0.45 \mu \mathrm{m}$ ) and diluted. The TP concentration in the solution was determined by HPLC as described below.

\section{Preparation of TP ethosomes and liposomes}

TP ethosomes were prepared as previously reported and consisted of 3\% PC, $40 \%$ ethanol, $0.75 \%$ TP, $0.5 \%$ CTAB, $0.5 \%$ Cremophor EL-35, and triple-distilled water to $100 \%(\mathrm{w} / \mathrm{w})$. Typically, PC was poured into a glass vial and dissolved along with TP and Cremophor EL-35 in ethanol. The glass vial was hermetically sealed and connected with a Teflon tube to a syringe system to allow the addition of the water phase and to avoid, as much as possible, ethanol evaporation. CTAB was dissolved along with triple-distilled water and was added in small increments with constant mixing. The preparation was mixed with a RET control/t (IKA, Staufen, Germany) at 700 rpm for 15 minutes at $30^{\circ} \mathrm{C} \pm 2^{\circ} \mathrm{C}$ and then left to cool at room temperature. All the procedures for the ethosomes preparation were carried out under a nitrogen atmosphere. Ethosomes were sterilized by extrusion through a $0.45 \mu \mathrm{m}$ sterile filter.

TP liposomes were prepared by the method described previously, with slight modifications. ${ }^{17,18}$ Three percent PC, $0.6 \%$ cholesterol, $0.75 \%$ TP and $0.5 \%$ Cremophor EL-35 were dissolved with ethanol as the lipid phase. The lipid phase was added to deionized water containing $1 \% \mathrm{CTAB}$ to generate lipid dispersion with sufficient stirring at $70^{\circ} \mathrm{C} \pm 2{ }^{\circ} \mathrm{C}$ until the ethanol scattered cleanly. The liposome suspension was further disrupted using an ultrasonic probe. The resulting liposomes were sterilized by extrusion through a $0.45 \mu \mathrm{m}$ sterile filter.

\section{TP vesicle characterization}

\section{Visualization by transmission electron microscopy}

The morphology of the ethosomes was observed using transmission electron microscopy (TEM). Briefly, a drop of the sample was applied to a film-coated copper grid. PTA solution was then dropped onto the grid. After drying, the specimen was viewed under a Philips TEM CM 12 electron 
microscope (Eindhoven, North Brabant, The Netherlands) at a 10,000-fold to 100,000-fold magnification.

\section{Vesicle size and zeta potential}

Particle size and zeta potential were measured by Zetasizer (ZetaPALS, Brookhaven, NY, USA). The polydispersity index (PDI) was used as a parameter of the size distribution. Before measurements, vesicular suspension was mixed with the appropriate medium. All particle sizes and zeta potential were measured at room temperature.

\section{Differential scanning calorimetry measurement}

The phase transition temperatures $\left(\mathrm{T}_{\mathrm{m}}\right)$ of the ethosomes and liposomes were measured using the Netzsch differential scanning calorimetry 200 F3 (Netzsch, Selb, Germany) equipped with a liquid nitrogen cooling system. The thermal analysis was performed at a temperature range of $-100^{\circ} \mathrm{C}$ to $80^{\circ} \mathrm{C}$, with a programmed heating rate of $10^{\circ} \mathrm{C} /$ minute, under a constant nitrogen stream. The $\mathrm{T}_{\mathrm{m}}$ values given are the temperatures recorded at the peak summit.

\section{Determination of vesicle entrapment efficiency}

The entrapment efficiency (EE\%) of TP vesicles was determined by the ultracentrifugation method. Briefly, vesicular suspensions were centrifuged at 50,000 rpm and $4^{\circ} \mathrm{C}$ for 1 hour (Optima Ultracentrifuge, Beckman Coulter, Fullerton, USA). Following centrifugation, the supernatant and vesicles were separated. The supernatant was removed and drug quantity was analyzed by HPLC. Meanwhile, an equal volume of vesicular suspension was used to assess the total amount of the drug $(\mathrm{T})$ which was measured after having dissolved vesicles in ethanol using an ultrasound bath for 10 minutes. The entrapment efficiency was calculated as follows: $(\mathrm{T}-\mathrm{C}) / \mathrm{T} \times 100 \%$, where $\mathrm{T}$ is the total amount of $\mathrm{TP}$ in the vesicle suspensions and $\mathrm{C}$ is the amount of drug detected only in the supernatant.

\section{Vesicle stability}

The stability of TP vesicles was determined by assessing the mean size and the entrapment efficiency of the vesicles over time. The measurements were conducted on three batches of ethosomes and liposomes that were kept at room temperature for different periods of time.

\section{HPLC analytical conditions for TP determination}

The HPLC system was carried out using a Waters apparatus equipped with a $2487 \mathrm{UV}$ detector and an Empower computerized analysis program. The assay was carried out at $240 \mathrm{~nm}$, using a NucleosilC ${ }_{18}$ column $(150 \times 4.6 \mathrm{~mm}$ inner diameter, $5 \mu \mathrm{m}$ ) at $35^{\circ} \mathrm{C}$, with a mobile phase composed of methanol: tetrahydrofuran: water $(53: 15: 32$, $\mathrm{v} / \mathrm{v} / \mathrm{v}$ ) at $1 \mathrm{~mL} / \mathrm{minute}$.

\section{Skin penetration and permeation studies} Skin permeation study of TP vesicles in vitro

All animal experiments were approved by the Institutional Animal Care and Use Committee of Liaoning Research Institute of Family Planning. Nine healthy male mice (weight, 18-22 g) were euthanized by $\mathrm{CO}_{2}$ inhalation and the abdominal hair was removed. After washing with distilled water, the abdominal skin was peeled off and the subcutaneous tissue and fat were removed. The intact mice skins were immersed in saline and stored in a refrigerator at $4^{\circ} \mathrm{C}$ until use within 24 hours.

The permeation of $5 \mathrm{~mL}$ TP vesicles vs $5 \mathrm{~mL}$ TP saturated solution in $40 \%$ ethanol was carried out in side-by-side diffusion cells (Valia-Chien chambers) through the abdominal skin of male mice for 18 hours at $32^{\circ} \mathrm{C} \pm 1^{\circ} \mathrm{C}$. The samples were added in a nonocclusive manner into a $1.33 \mathrm{~cm}^{2}$ diffusion area. The receiver medium contained $6 \mathrm{~mL} \mathrm{3 \%}$ Cremophor EL-35 aqueous solution and was constantly stirred by magnetic stirrer at $100 \mathrm{rpm}$. In order to ensure pseudosink conditions, $6 \mathrm{~mL}$ of receiver medium was withdrawn at 2 -hour intervals. For each sample removed from the receiver, the same volume of fresh diffusion medium was added. The solution collected was filtered through a $0.45 \mu \mathrm{m}$ microporous membrane. The filtrate was quantified by HPLC. The cumulative amount of drug was calculated according to the following formula: $\mathrm{Q}_{\mathrm{n}}=\sum \mathrm{C}_{\mathrm{i}} \mathrm{V} / \mathrm{A}$. The cumulative amount of drug that permeated per unit area was plotted across time. The steady-state permeation rate $\left(\mathrm{J}_{\mathrm{s}}, \mu \mathrm{g} / \mathrm{cm}^{2} / \mathrm{hour}\right)$ and lag time were calculated from the slope and $\mathrm{x}$-intercept of the linear portion of the graph, respectively.

\section{Depth of RR skin penetration from vesicles} visualized by confocal laser scanning microscopy The depth and mechanism of skin penetration of RR-loaded vesicles were investigated using confocal laser scanning microscopy (CLSM). The probe-loaded vesicles were applied via a Hill Top chamber (Hill Top Research Inc, Ohio, USA) for 8 hours to the dorsal skin of rats. Treated rats were kept in a separate cage and maintained under laboratory conditions. Food and water were allowed ad libitum. The rat was sacrificed by heart puncture, and the dorsal skin was excised and washed with distilled water. The skin was sectioned into $1 \mathrm{~mm}^{2}$ pieces 
and evaluated for the depth of the probe penetration. The skin thickness was optically scanned at $20 \mu \mathrm{m}$ increments through the $\mathrm{z}$-axis of a Leica TCS-sp5 confocal laser scanning microscope (Leica, Heidelberg, Germany). Optical excitation was carried out with a $488 \mathrm{~nm}$ Argon laser beam, and fluorescence emission of RR was detected above $560 \mathrm{~nm}$.

\section{In vivo study of TP transdermal system} and measurement of serum concentration

In vivo experiments with TP vesicles were carried out in 9 male SD rats $(240-270 \mathrm{~g})$ that were divided into three groups. The rats were castrated under anesthesia after acclimation. A 2-week recovery period was allowed for baseline testosterone to drop below $0.4 \mathrm{ng} / \mathrm{mL}$. On the day prior to the experiment, blood samples $(500 \mu \mathrm{L})$ were withdrawn to assay the baseline levels of testosterone to evaluate the efficiency of the castration. Then a $25 \mathrm{~cm}^{2}$ application area was shaved on the rat dorsal skin. On the day of the experiment, the animals were anesthetized by ether. The TP ethosomal and liposomal vesicle formulations (containing $2 \mathrm{mg}$ of TP) and 40\% ethanol saturated solution were applied to the rat shaved dorsal skin using a Hill Top chamber. Blood samples of $400 \mu \mathrm{L}$ were taken at $3,6,9,12,24,36$, and 48 hours via the retroorbital sinus after application. The samples were collected into nonheparinized vials, left standing for 0.5 hours, and centrifuged for serum separation. The separated serum was frozen at $-20^{\circ} \mathrm{C}$ until analysis. The drug concentration was measured by a Cobase 411 analyzer (Roche, Mannheim, Germany). On the basis of the testosterone serum concentrations, the following pharmacokinetic parameters were obtained.

- $\mathrm{C}_{\max }$ is the peak serum concentration during the dosing period.

- $\mathrm{AUC}$ is the area under the serum concentration-time curve calculated by the trapezoidal rule for a period from 0 to 48 hours.

\section{Statistical analysis}

All data are expressed as mean \pm SD. Statistical analysis was checked by the Student's $t$-test using the software SPSS 13.0 (IBM Corporation, Armonk, NY, USA). A value of $P<0.05$ was considered statistically significant.

\section{Results}

\section{TP solubility}

Different concentrations of surfactants were added to the system to increase the solubility of TP because of the
Table I Solubility of TP in various receiver solutions at $37^{\circ} \mathrm{C}$

\begin{tabular}{lcll}
\hline Solvent & $\begin{array}{l}\text { Solubility } \\
(\mu \mathrm{g} / \mathrm{mL})\end{array}$ & Solvent & $\begin{array}{l}\text { Solubility } \\
(\mu \mathrm{g} / \mathrm{mL})\end{array}$ \\
\hline $\mathrm{H}_{2} \mathrm{O}$ & \multicolumn{1}{c}{0} & $0.5 \% \mathrm{EL}-35 / \mathrm{H}_{2} \mathrm{O}$ & $70.5 \mathrm{I} \pm 2.30$ \\
$0.01 \% \mathrm{SDS} / \mathrm{H}_{2} \mathrm{O}$ & $0.22 \pm 0.0 \mathrm{I}$ & $1 \% \mathrm{EL}-35 / \mathrm{H}_{2} \mathrm{O}$ & $131.82 \pm 5.5 \mathrm{I}$ \\
$0.05 \% \mathrm{SDS} / \mathrm{H}_{2} \mathrm{O}$ & $1.85 \pm 0.05$ & $2 \% \mathrm{EL}-35 / \mathrm{H}_{2} \mathrm{O}$ & $274.85 \pm 4.8 \mathrm{I}$ \\
$0.1 \% \mathrm{SDS} / \mathrm{H}_{2} \mathrm{O}$ & $9.04 \pm 0.13$ & $3 \% \mathrm{EL}-35 / \mathrm{H}_{2} \mathrm{O}$ & $383.89 \pm 15.4 \mathrm{I}$ \\
$0.2 \% \mathrm{SDS} / \mathrm{H}_{2} \mathrm{O}$ & $123.38 \pm 9.4$ & $1 \%$ Tween-80/ $\mathrm{H}_{2} \mathrm{O}$ & $102.72 \pm 8.54$ \\
$5 \% \mathrm{PEG} 400 / \mathrm{H}_{2} \mathrm{O}$ & $2.13 \pm 0.04$ & $3 \%$ Tween-80/ $/ \mathrm{H}_{2} \mathrm{O}$ & $327.95 \pm 19.45$ \\
$10 \% \mathrm{PEG} 400 / \mathrm{H}_{2} \mathrm{O}$ & $6.18 \pm 0.27$ & $5 \%$ Tween-80/ $\mathrm{H}_{2} \mathrm{O}$ & $371.1 \mathrm{I} \pm 14.90$ \\
$20 \% \mathrm{PEG} 400 / \mathrm{H}_{2} \mathrm{O}$ & $10.83 \pm 0.43$ & $10 \%$ Tween-80/ $\mathrm{H}_{2} \mathrm{O}$ & $432.53 \pm 20.87$ \\
$30 \% \mathrm{PEG} 400 / \mathrm{H}_{2} \mathrm{O}$ & $20.76 \pm 1.69$ & & \\
\hline
\end{tabular}

Note: Each value represents the mean $\pm S D(n=3)$.

Abbreviations: EL-35, Cremophor EL-35; PEG400, polyethylene glycol-400; SD, standard deviation; SDS, sodium dodecyl sulfate; TP, testosterone propionate.

insolubility of TP in water. The results are presented in Table 1, which reveals that the TP solubility was the highest when the surfactant used was 3\% Cremophor EL-35 aqueous solution. Therefore, a 3\% Cremophor EL-35 aqueous solution was selected as the receiver medium for the skin permeation study.

\section{Preparation of TP ethosomes and liposomes}

The effects of CTAB, Cremophor EL-35, and TP on the particle size and zeta potential of the vesicles were investigated at 3\% PC and 40\% ethanol. The mean size distribution decreased rapidly and the zeta potential of the ethosomes increased when the concentration of CTAB ranged from $0 \%$ to $1 \% \mathrm{w} / \mathrm{w}$. It is noteworthy that only $0.2 \% \mathrm{w} / \mathrm{w}$ CTAB

Table 2 Physicochemical characteristics of ethosomes prepared with different concentration of CTAB, TP and EL-35

\begin{tabular}{lllrr}
\hline Composition & $\begin{array}{l}\text { Concentration } \\
(\% \mathbf{w} / \mathbf{w})\end{array}$ & Size $(\mathbf{n m})$ & $\begin{array}{l}\text { PDI } \\
\end{array}$ & \multicolumn{1}{l}{$\begin{array}{l}\text { Zeta } \\
\text { potential } \\
(\mathbf{m V})\end{array}$} \\
\hline CTAB & 0 & $647.3 \pm 40.2$ & 0.386 & $-1.22 \pm 0.89$ \\
& 0.2 & $266.4 \pm 1.4$ & 0.100 & $9.39 \pm 1.00$ \\
& 0.5 & $183.6 \pm 2.4$ & 0.096 & $10.58 \pm 0.88$ \\
& 1 & $150.2 \pm 0.7$ & 0.096 & $11.43 \pm 1.05$ \\
TP & 0 & $160.3 \pm 3.5$ & 0.092 & $5.95 \pm 0.79$ \\
& 0.5 & $175.8 \pm 2.4$ & 0.087 & $13.68 \pm 0.51$ \\
& 0.75 & $156.5 \pm 3.1$ & 0.096 & $11.73 \pm 0.54$ \\
& 1 & $151.5 \pm 2.2$ & 0.115 & $8.01 \pm 1.17$ \\
EL-35 & 0 & $203.9 \pm 10.6$ & 0.099 & $10.76 \pm 0.14$ \\
& 0.5 & $192.1 \pm 7.2$ & 0.085 & $13.81 \pm 0.09$ \\
& 1 & $179.8 \pm 13.2$ & 0.155 & $13.40 \pm 0.94$ \\
& 1.5 & $178.5 \pm 8.1$ & 0.110 & $12.43 \pm 0.33$ \\
\hline
\end{tabular}

Note: Each value represents the mean \pm SD $(n=3)$.

Abbreviations: CTAB, hexadecyl trimethyl ammonium bromide; EL-35, Cremophor EL-35; PDI, polydispersity index; SD, standard deviation; TP, testosterone propionate. 
could lead to a transition of the charge of the ethosomes from negative $(-1.22 \pm 0.89 \mathrm{mV})$ to positive $(9.39 \pm 1.00 \mathrm{mV})$ (Table 2). When the TP concentration was elevated from $0 \%$ to $1 \% \mathrm{w} / \mathrm{w}$, and other ratios were unchanged (Table 2), the mean size distribution of empty vesicles was $160.3 \pm 3.5 \mathrm{~nm}$, $\mathrm{PDI}=0.092$. No change in the vesicle size was observed following the addition of $1 \% \mathrm{TP}$ to the system $(151.5 \pm 2.2 \mathrm{~nm}$, PDI $=0.115)$. When the Cremophor EL-35 concentration was elevated from $0 \%$ to $1.5 \% \mathrm{w} / \mathrm{w}$, and other ratios were unchanged (Table 2), the mean size distribution slowly decreased, and drug loading ability increased. The size distribution of the TP ethosomes shows one narrow peak, indicating that the vesicle population is relatively homogenous in size.

All in all, the optimum composition of the TP ethosome is as follows: $3 \% \mathrm{PC}, 40 \%$ ethanol, $0.75 \% \mathrm{TP}, 0.5 \% \mathrm{CTAB}$, $0.5 \%$ Cremophor EL-35, and $\mathrm{H}_{2} \mathrm{O}$ to $100 \%$. The optimum composition of the TP liposome is as follows: $3 \% \mathrm{PC}, 0.6 \%$ cholesterol, $0.75 \%$ TP, $1.0 \%$ CTAB, $0.5 \%$ Cremophor EL-35 and $93.75 \% \mathrm{H}_{2} \mathrm{O}$. The particle size, $\mathrm{PDI}$, and zeta potential of TP liposome for three batches were $174.4 \pm 16.5 \mathrm{~nm}, 0.176$, and $39.64 \pm 3.38 \mathrm{mV}$, respectively.

\section{Characterization of TP vesicles}

\section{Structure}

Micrographs of TP ethosomes negatively stained by PTA showed that they were unilamellar vesicles with a closed spherical shape (Figure 1). These micrographs confirmed that TP ethosomes have only a few lamellae.

\section{Calorimetry of vesicular systems}

The melting temperatures of the vesicular systems were evaluated as a measure of vesicle softness. The results of

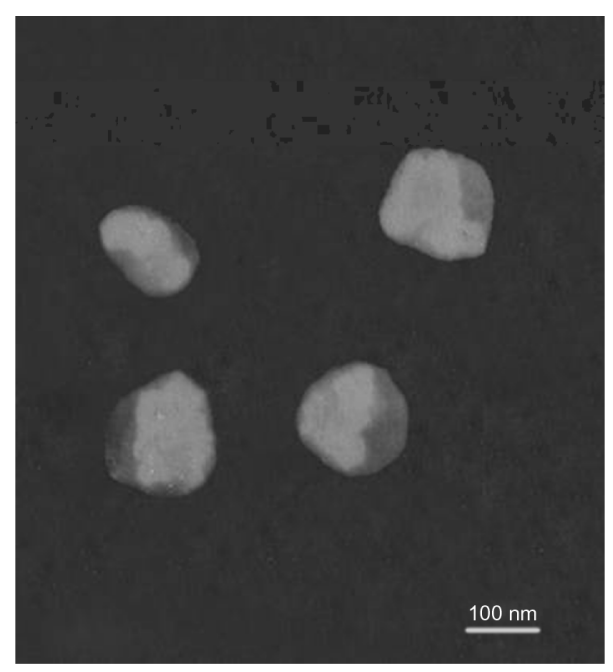

Figure I Microphotographs of TP ethosomes by TEM (scale bar, $100 \mathrm{~nm}$ ). Abbreviations: TP, testosterone propionate; TEM, transmission electron microscopy.

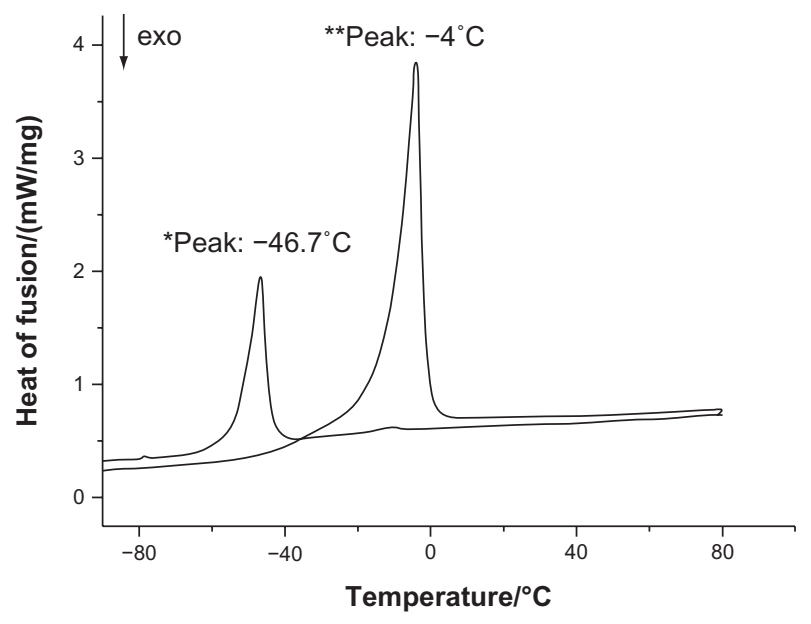

Figure 2 DSC of TP ethosomes* and liposomes**.

Abbreviations: DSC, differential scanning calorimetry; TP, testosterone propionate.

the analysis showed a melting temperature of $-46.7^{\circ} \mathrm{C}$ and $-4.0^{\circ} \mathrm{C}$ for TP ethosomes and liposomes, respectively (Figure $2 \mathrm{TP}$ ethosomes*and liposomes**).

\section{Entrapment efficiency of TP vesicles}

The entrapment efficiency of the vesicles was determined by the ultracentrifugation method. The results indicate that the TP entrapment efficiency in ethosomes was very high, with values of $92.7 \% \pm 3.7 \%$. However, the entrapment efficiency of TP liposomes was only $64.7 \% \pm 2.1 \%$. The result showed that the entrapment efficiency of the TP ethosomes was significantly higher $(P<0.05)$ than that of liposomes.

\section{Stability of TP vesicles}

Figures 3 and 4 present the EE\% and mean size of vesicles, respectively, that were kept at room temperature and measured at various time intervals following vesicle preparation. Although there is a linear increase in particle size across time, the analytical results indicate that the mean size and $\mathrm{EE} \%$ of the ethosomes did not change significantly $(P>0.05)$ over time. However, the change in EE\% of TP liposomes across time was found to be statistically $(P<0.05)$ significant, while the change in particle size was statistically insignificant $(P>0.05)$. TEM visualization of ethosomes supports these results and indicates that no significant structural change of the vesicles occurs over time.

\section{Penetration and permeation through the skin TP skin permeation}

The skin permeation of TP through male mouse skin was determined in side-by-side diffusion cells from three 


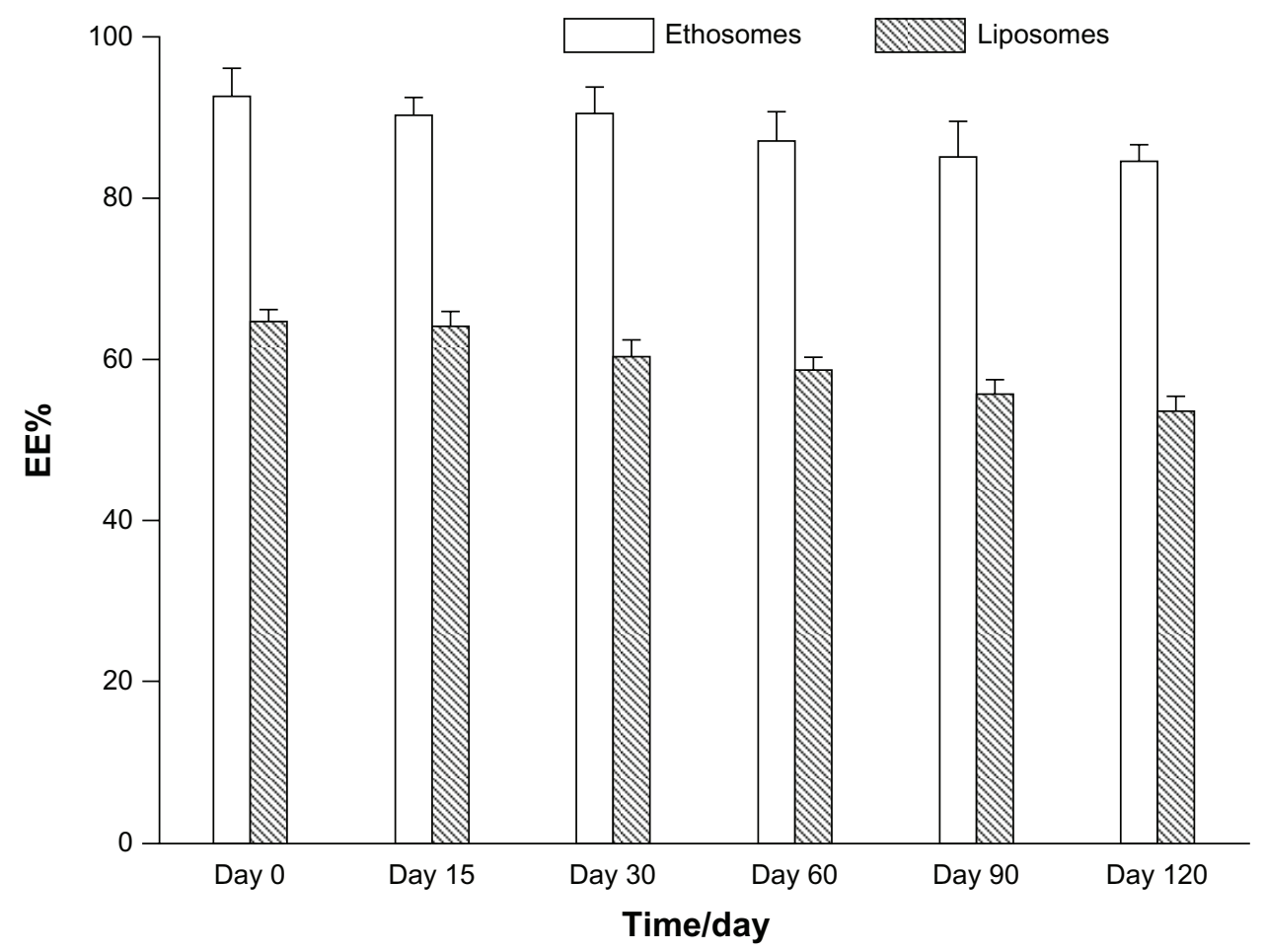

Figure 3 The change in the entrapment efficiency of the TP ethosomes and liposomes over time. Note: Each data point represents the mean \pm SD $(n=3)$.

Abbreviations: $\mathrm{EE}$, entrapment efficiency; SD, standard deviation; TP, testosterone propionate.

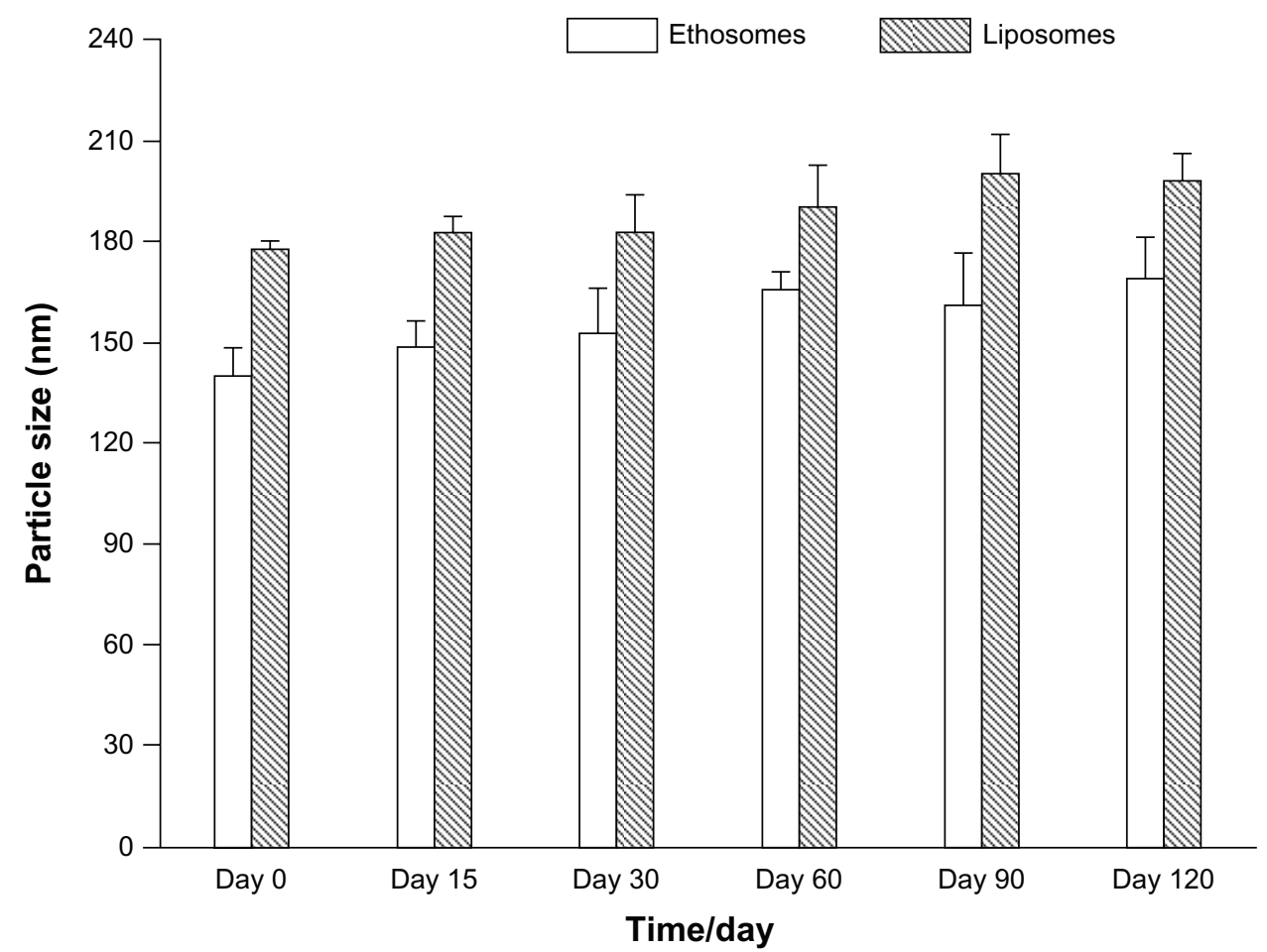

Figure 4 The change in the mean particle size of the TP ethosomes and liposomes over time. Note: Each data point represents the mean \pm SD $(n=3)$.

Abbreviations: SD, standard deviation; TP, testosterone propionate. 


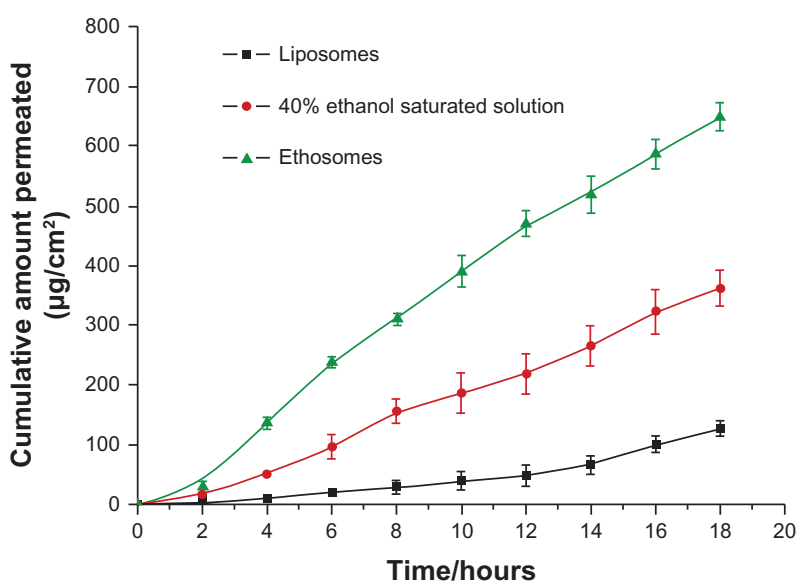

Figure 5 TP skin permeation profiles through mice skin from vesicular formulation versus ethanol-saturated solution in vitro.

Note: Each data point represents the mean \pm SD $(n=3)$.

Abbreviations: SD, standard deviation; TP, testosterone propionate.

systems, each containing $0.75 \%$ TP ethosomes, liposomes, or $40 \%$ hydroethanolic saturation solution. The timedependent permeation of TP systems is shown in Figure 5. It can be seen from these plots that ethosomes increased the amount of TP that permeated the skin over time relative to liposomes and the $40 \%$ hydroethanolic saturation solution.

The value of steady-state transdermal flux for TP ethosomes was calculated as $37.85 \pm 2.8 \mu \mathrm{g} / \mathrm{cm}^{2} /$ hour, with a lag time of 0.18 hours. The $40 \%$ hydroethanolic saturation solution and liposomes provided significantly lower $(P<0.05)$ flux values and longer lag times of $21.72 \pm 3.6 \mu \mathrm{g} / \mathrm{cm}^{2} /$ hour, 1.4 hours and $7.30 \pm 1.4 \mu \mathrm{g} / \mathrm{cm}^{2} /$ hour, 3.2 hours, respectively. The results show that the ethosome formulation could enhance the transdermal permeation of TP by fivefold compared with the liposome formulation.

\section{CLSM determination of TP vesicular transdermal delivery}

The lipophilic fluorescent probe rhodamine red was applied to study skin penetration from TP vesicles into rat skin using a Hill Top chamber. As shown in Figure 6, CLSM demonstrated that the TP liposomes did not facilitate probe penetration into the skin. Instead, the application of the TP liposomes resulted in only a small reservoir in the upper layers of skin (up to about $120 \mu \mathrm{m}$ ). Using the TP hydroethanolic solution, a relatively deep penetration (up to about $160 \mu \mathrm{m}$ ) was measured, but with a relatively very low fluorescence intensity. The use of the ethosomal system resulted in an increase in both depth of penetration (up to about $260 \mu \mathrm{m}$ ) and fluorescence intensity.

\section{In vivo studies of the TP transdermal system}

The results of the analysis of the blood samples show that $\mathrm{C}_{\text {max }}$ and AUC values for the ethosomal system were significantly higher $(P<0.05)$ compared with the TP liposomes. In the pharmacokinetic experiments, the serum concentration of TP from the ethosomal system was high and stable after 5 hours, as shown in Figure 7. The pharmacokinetic results for the three groups were consistent with the onecompartment model. The value of AUC was $160.39 \mathrm{ng} /$ $\mathrm{mL} \cdot$ hours in the TP ethosomal system, $65.84 \mathrm{ng} / \mathrm{mL} \cdot$ hours in the TP $40 \%$ hydroethanolic solution system, and $32.40 \mathrm{ng} /$ $\mathrm{mL}$.hours in the TP liposomal system. The AUC value of the TP ethosomal system was 2.43 times greater than that of the TP 40\% hydroethanolic solution system and 4.95 times greater than that of the TP liposomal system. The $\mathrm{C}_{\max }$ of the three systems was $9.75 \mathrm{ng} / \mathrm{mL}, 3.76 \mathrm{ng} / \mathrm{mL}$, and $2.29 \mathrm{ng} / \mathrm{mL}$, respectively. These results demonstrated that TP ethosomes could improve drug absorption and bioavailability compared with the other two preparations. In addition, the $T_{1 / 2 \beta}$ value
A

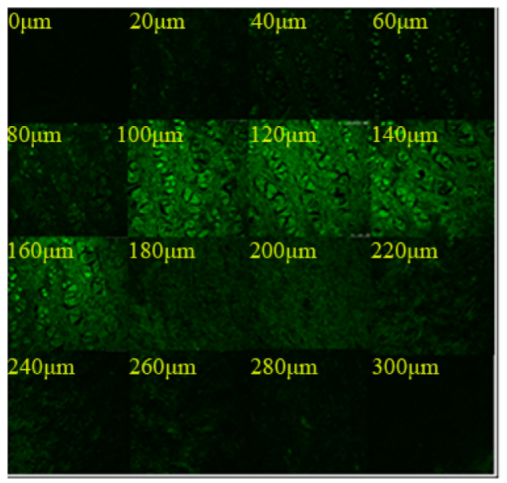

B

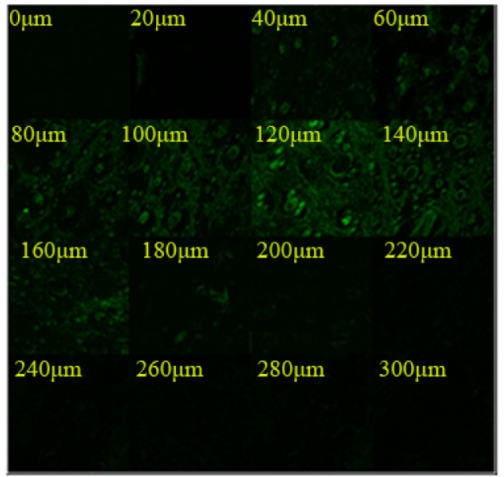

C

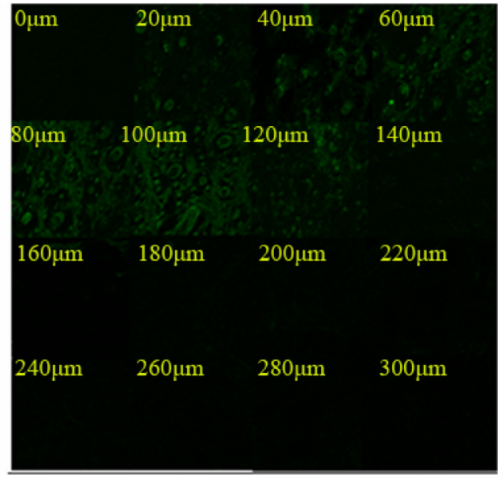

Figure 6 CLSM micrographs of mouse skin after application of the fluorescent probe RR in TP ethosomes (A), TP hydroethanolic solution (B), and TP liposomes (C). Abbreviations: CLSM, confocal laser scanning microscopy; RR, rhodamine red-X and succinimidyl ester; TP, testosterone propionate. 


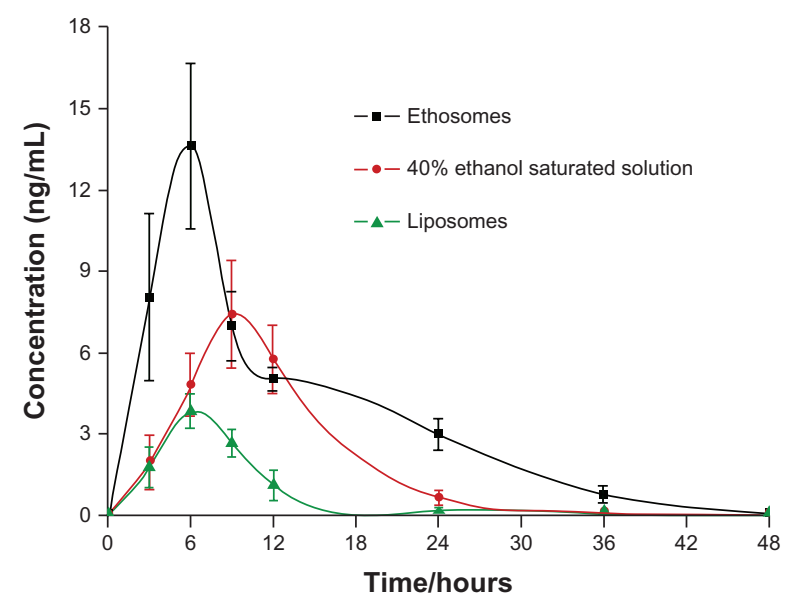

Figure 7 Serum concentration-time curve of three TP preparations. Note: Each data point represents the mean $\pm S D(n=3)$.

Abbreviations: SD, standard deviation; TP, testosterone propionate.

was 7.34 hours in TP ethosomes, 4.67 hours in TP liposomes, and 4.89 hours in the TP $40 \%$ hydroethanolic solution. Compared with the other two preparations, TP ethosomes have a significantly longer $(P<0.05)$ elimination half-life and more stable plasma concentrations at a high level. Therefore, TP ethosomes have an obvious advantage compared with the other two preparations.

\section{Discussion TP solubility}

The solubility of a drug in the vesicular system can influence its entrapment efficiency, vesicular stability, and permeation through skin. ${ }^{19} \mathrm{TP}$ is a lipophilic molecule, so water is not suitable as a receiver medium. Ethanol is known for its ability to fluidize lipids in the stratum corneum of the skin, so it is possible that it could enhance the skin penetration of molecules when used as a receiver medium. ${ }^{20}$ Therefore, it is very important to select an appropriate receiver medium for in vitro permeation experiments, which permit the maintenance of sink conditions throughout the experiment. Based on the TP solubility results, a 3\% Cremophor EL-35 aqueous solution was used for the receiver medium.

\section{Preparation}

Ethosomes are novel permeation-enhancing lipid vesicles that contain a high concentration of ethanol. During the preparation, we found that the particle size of the ethosomes decreased as the ethanol concentration increased, but increased as the phospholipid concentration increased. Ethanol could increase the solubility of TP, but too high an ethanol concentration is not conducive to ethosomes formation because phospholipids can easily dissolve in ethanol.
Therefore, the ethanol concentration in the ethosomes system should be kept at $25 \%-45 \%$.

CTAB is a cationic surfactant. The CTAB concentration is an important factor in the control of the particle size and zeta potential of ethosomes. In the process of preparing ethosomes, the sample was stratified without CTAB when it was placed at room temperature for even a brief period. Moreover, CTAB makes the ethosomal vesicles positively charged. The charge of the vesicles is an important characteristic that can influence vesicular properties. Due to electrostatic repulsion, it can reduce the mutual aggregation and fusion of vesicles and increase the stability of ethosomes. Ethosomes without CTAB exhibited a zeta potential of $-1.22 \pm 0.89 \mathrm{mV}$, and the addition of $0.5 \%$ CTAB induced a transition in the charge of the vesicles from negative to positive $(10.58 \pm 0.88 \mathrm{mV})$. The negative charge of the skin surface and affinity between positive and negative charges therefore results in increased permeation of the drug.

Cremophor EL-35 is a nonionic surfactant. Cremophor EL-35 in an ethosomal system may increase TP solubility and drug loading ability. In the stability study, TP slowly precipitated out as the Cremophor EL-35 concentration became $<0.5 \%$.

\section{Calorimetry of vesicular systems}

Compared with liposomes, ethosomes that contain ethanol have a lower melting temperature. The presence of ethanol increases the flexibility of the lipid bilayer in the ethosomes. Furthermore, the interaction between ethanol and the lipid of the stratum corneum reduces the phase transition temperature of the ethosomes, thus promoting mobility and drug penetration. The results showed that the lower the $T_{m}$ of the sample, the better the mobility and transmission of the sample.

\section{Entrapment efficiency of TP vesicles}

The entrapment efficiency of the ethosomes was significantly higher than that of conventional vesicle preparations. ${ }^{21}$ There are three factors controlling the entrapment efficiency of the vesicles. The first is the ethanol concentration. Compared with liposomes, the most striking feature of ethosomes is the relatively high concentration of ethanol. Barry demonstrated that ethanol binds strongly to the bilayers in the region of the lipid-water interface with little or no interaction with the hydrocarbon interior. ${ }^{22}$. The second factor is the presence of surfactants, which contribute to vesicle stability and reduce the likelihood of vesicle leakage. The last factor is the characteristics of the 
drug. In general, lipophilic drugs are easier to entrap than hydrophilic drugs. ${ }^{23 .}$

\section{Penetration and permeation through the skin}

In spite of several advantages offered by transdermal delivery, only a few molecules actually pass through the skin because of the stratum corneum barrier. To improve the permeability of the drug, specially designed vesicles were proposed to allow transdermal delivery, including elastic liposomes, ethosomes, and so on. ${ }^{24-27}$

The in vitro release rates and cumulative amount delivered are regarded as an important index of quality for a transdermal drug delivery system. According to the test results, a TP flux from the ethosomal formulation through mouse skin of $37.85 \pm 2.8 \mu \mathrm{g} / \mathrm{cm}^{2} /$ hour was calculated using the transdermal program. The amount of TP that permeated the skin after 18 hours from the ethosomes was higher $(P<0.05)$ than the amount from liposomes $\left(650.24 \pm 39.9 \mu \mathrm{g} / \mathrm{cm}^{2}\right.$ and $127.29 \pm 23.0 \mu \mathrm{g} / \mathrm{cm}^{2}$, respectively). The results show that ethosomes increased transdermal permeation of TP by fivefold. This can be explained by the fact that ethanol has permeation-enhancing properties. Ethanol is very efficient in enhancing the skin permeation of a number of drugs; it has been shown that the physicochemical characteristics of ethosomes allow this vesicular carrier to transport active substances more efficaciously through the stratum corneum into the deeper layers of the skin than liposomes. ${ }^{12}$ Ethanol can increase the solubility of the drug in the vehicle, alter the solubility properties of the tissue, and even extract some of the lipid fraction from within the stratum corneum. ${ }^{22}$ Using the fluorescent probe RR, we were able to visualize the penetration of the molecule from various carriers into the skin. As expected, penetration from TP liposomes was only through the upper layers of the skin. Moreover, the amount of probe visualized was very small. In contrast, enhanced delivery of RR, in terms of depth and quantity, was observed using the ethosomal carrier. While the TP $40 \%$ hydroalcoholic solution was able to facilitate penetration of RR deeply into the skin, the amount delivered was small relative to the delivery from the ethosomal system. Ethosomes were able to enhance the penetration of a relatively large quantity of the probe deeply into the skin. In vivo studies of the TP transdermal system, the AUC of the TP ethosome group was fivefold greater than that of the liposome group, and twofold greater than that of the hydroalcoholic solution group. These results indicate that TP ethosomes may play an important role in promoting drug absorption and application compared with the other two preparations.

The transdermal delivery of testosterone is the most efficient and convenient way of administration for the advantage of its mimicry close to the circadian pattern of healthy men; it has also been widely prescribed for postmenopausal women in the United States. ${ }^{28}$ To date, there are many testosterone transdermal formulations in use in clinics, including Testoderm, Androderm, Testim, AndroGel, as well as Intrinsa. Intrinsa is a testosterone patch to be used for the treatment of hypoactive sexual desire disorder in menopausal women. Long-term use of these patches over the last 3 to 10 years has been demonstrated to be effective in maintaining sexual function and bone and muscle mass. The major drawbacks, including itching, irritation, and burn-like blisters or redness at the application site, limit the applications of the transdermal patches in clinics. Although testosterone gel causes only minimal skin irritation, it must be applied to such a large surface area of the skin $\left(>400 \mathrm{~cm}^{2}\right)$ that it may not be acceptable to all patients. The goal of our study was to achieve better testosterone skin permeation, maintaining a higher testosterone blood level with a smaller application surface area and minimal skin irritation. The surfactant-modified TP ethosomal system provided an appropriate approach for the transdermal delivery of TP. Particle size and zeta potential were modified by surfactants, while entrapment efficiency and penetration of TP ethosomes are significantly higher than that of literature reported. ${ }^{16}$ Moreover, the half-life of TP is longer than that of testosterone. TP ethosomal application can reduce the administration time because of its slow metabolism and extended pharmacological action after it is absorbed.

\section{Conclusion}

In summary, the surfactant-modified TP ethosomes showed higher entrapment efficiency and were relatively more stable than other liposomal formulations because ethanol can efficiently entrap the drug. Delivery through the ethosomal system indicated an enhanced transdermal flux and lower lag time than the other systems tested. The CLSM results showed that the penetration ability of the ethosomal formulation was higher than that of the liposomal formulation in term of RR intensity in the rat skin. Therefore, the ethosomal system could provide an appropriate approach for the transdermal delivery of TP.

\section{Acknowledgments}

This work was supported by Liaoning Province Natural Science Fund (20091036). The authors thank the Liaoning 
University of Traditional Chinese Medicine, Shenyang, People's Republic of China, for providing assistance in CLSM.

\section{Disclosure}

The authors report no conflicts of interest in this work.

\section{References}

1. Bagatell CJ, Bremner WJ. Androgens in men - uses and abuses. NEngl J Med. 1996;334(11):707-714.

2. Basaria S, Dobs AS. Hypogonadism and androgen replacement therapy in elderly men. Am J Med 2001;110(7):563-572.

3. Yassin AA, Haffejee M. Testosterone depot injection in male hypogonadism: a critical appraisal. Clin Interv Aging. 2007;2(4):577-590.

4. Tan RS, Culberson JW. An integrative review on current evidence of testosterone replacement therapy for the andropause. Maturitas. 2003;45(1):15-27.

5. Nieschlag E, Büchter D, Von Eckardstein S, Abshagen K, Simoni M, Behre HM. Repeated intramuscular injections of testosterone undecanoate for substitution therapy in hypogonadal men. Clin Endocrinol (Oxf). 1999;51(6):757-763.

6. Behre HM, Wang C, Handelsman DJ, Nieschlag E. Pharmacology of testosterone preparations. In: Neischlag E, Behre HM, editors. Testosterone: Action, Deficiency, Substitution. Cambridge: Cambridge University Press; 2004:405-445.

7. Kaplun-FrischoffY, Touitou E. Testosterone skin permeation enhancement by menthol through formation of eutectic with drug and interaction with skin lipids. J Pharm Sci. 1997;86(12):1394-1399.

8. Dayan N, Touitou E. Carriers for skin delivery of trihexyphenidyl $\mathrm{HCl}$ : ethosomes vs liposomes. Biomaterials. 2000;21(18):1879-1885.

9. Godin B, Touitou E. Mechanism of bacitracin permeation enhancement through the skin and cellular membranes from an ethosomal carrier. J Control Release. 2004;94(2-3):365-379.

10. Paolino D, Lucania G, Mardente D, Alhaique F, Fresta M. Ethosomes for skin delivery of ammonium glycyrrhizinate: in vitro percutaneous permeation through human skin and in vivo anti-inflammatory activity on human volunteers. J Control Release. 2005;106(1-2):99-110.

11. Elsayed MM, Abdallah OY, Naggar VF, Khalafallah NM. Deformable liposomes and ethosomes: mechanism of enhanced skin delivery. Int $J$ Pharm. 2006;322(1-2):60-66.

12. Touitou E, Dayan N, Bergelson L, Godin B, Eliaz M. Ethosomes - novel vesicular carriers for enhanced delivery: characterization and skin penetration properties. J Control Release. 2000;65(3): 403-418.
13. Jain HP, J. Joshi, K. Patel, P. Upadhyay, U.M. . ethosome: a novel drug carrier. International journal of comprehensive pharmacy. 2011;7(1):1-4.

14. Touitou E, Godin, B. Weiss, C. Enhanced delivery of drugs into and across the skin by ethosomal carriers. Drug Dev Res. 2000;50:(3-4)406-415.

15. Dubey V, Mishra D, Jain NK. Melatonin loaded ethanolic liposomes: physicochemical characterization and enhanced transdermal delivery. Eur J Pharm Biopharm. 2007;67(2):398-405.

16. Ainbinder D, Touitou E. Testosterone ethosomes for enhanced transdermal delivery. Drug Deliv. 2005;12(5):297-303.

17. Yang S, Chen J, Zhao D, Han D, Chen X. Comparative study on preparative methods of DC-Chol/DOPE liposomes and formulation optimization by determining encapsulation efficiency. Int $J$ Pharm. 2012;434(1-2):155-160.

18. Yu BT, Zhang ZR. Preparation and in vitro Drug-release Behavior of Andrographolide Liposomes. Chinese Journal of Pharmaceuticals 2009;40(2):114-116.

19. Bhatia A, Kumar R, Katare OP. Tamoxifen in topical liposomes: development, characterization and in-vitro evaluation. J Pharm Pharm Sci. 2004;7(2):252-259.

20. Williams AC, Barry BW. Penetration enhancers. Adv Drug Deliv Rev 2004;56(5):603-618

21. Rao Y, Zheng F, Zhang X, Gao J, Liang W. In vitro percutaneous permeation and skin accumulation of finasteride using vesicular ethosomal carriers. AAPS PharmSciTech. 2008;9(3):860-865.

22. Barry BW. Novel mechanisms and devices to enable successful transdermal drug delivery. Eur J Pharm Sci. 2001;14(2):101-114.

23. Barupal AK, Gupta V, Ramteke S. Preparation and Characterization of Ethosomes for Topical delivery of Aceclofenac. Indian J Pharm Sci. 2010;72(5):582-586.

24. Dubey V, Mishra D, Asthana A, Jain NK. Transdermal delivery of a pineal hormone: melatonin via elastic liposomes. Biomaterials. 2006;27(18):3491-3496.

25. Mishra D, Dubey V, Asthana A, Saraf DK, Jain NK. Elastic liposomes mediated transcutaneous immunization against Hepatitis B. Vaccine. 2006;24(22):4847-4855.

26. Jain S, Jain N, Bhadra D, Tiwary AK, Jain NK. Transdermal delivery of an analgesic agent using elastic liposomes: preparation, characterization and performance evaluation. Curr Drug Deliv. 2005;2(3):223-233.

27. Elsayed MM, Abdallah OY, Naggar VF, Khalafallah NM. Lipid vesicles for skin delivery of drugs: reviewing three decades of research. Int $J$ Pharm. 2007;332(1-2):1-16.

28. Snabes MC, Simes SM. Approved hormonal treatments for HSDD: an unmet medical need. $J$ Sex Med. 2009;6(7):1846-1849.
International Journal of Nanomedicine

\section{Publish your work in this journal}

The International Journal of Nanomedicine is an international, peerreviewed journal focusing on the application of nanotechnology in diagnostics, therapeutics, and drug delivery systems throughout the biomedical field. This journal is indexed on PubMed Central, MedLine, CAS, SciSearch ${ }^{\circledR}$, Current Contents ${ }^{\circledR} /$ Clinical Medicine,

\section{Dovepress}

Journal Citation Reports/Science Edition, EMBase, Scopus and the Elsevier Bibliographic databases. The manuscript management system is completely online and includes a very quick and fair peer-review system, which is all easy to use. Visit http://www.dovepress.com/ testimonials.php to read real quotes from published authors. 